\title{
Development of a Micro-scale Assembly Facility with a Three Fingered, Self-aware Assembly Tool and Electro-chemical Etching Capabilities
}

\author{
Jacob W. Chesna ${ }^{1}$, Stuart T. Smith ${ }^{1}$, D.J. Hastings ${ }^{1}$, Borja de la Maza ${ }^{2}$, \\ Bartoz K. Nowakowski ${ }^{1}$, and Feilong Lin $^{1}$ \\ ${ }^{1}$ Center for Precision Metrology at the University of North Carolina at Charlotte \\ NC 28223, USA \\ ${ }^{2}$ Innovalia Metrology, Rodríguez arias 6, 48008, Bilbao-Vizcaya, Spain
}

\begin{abstract}
This abstract outlines current developments of a microassembly facility focusing on studies using a three fingered gripper. Individual fingers of the gripper comprise thin fibers ranging in diameter from 7 to $80 \mathrm{~m}$ that are attached to quartz based oscillators and are capable of sensing proximity, contact, and controlling adhesion forces. To optimize gripper finger performance, an electrolytic etching facility has been used to selectively modify oscillator performance and this system is currently being developed for automated processing. To demonstrate current performance of the gripper system, a micro-CMM contact sensing probe has been assembled and a preliminary performance evaluation is presented.
\end{abstract}

Keywords: Micro-robotics, 3D surface profilometry, micro-scale, high aspect ratio, standing wave probes, micro-CMM.

\section{Introduction}

Over the past decade the research group in the Center for Precision Metrology at the University of North Carolina at Charlotte has developed a series of microscale devices that focus around micro-assembly machinery and sensing devices for force and position feedback. Design elements from many projects have been collectively used in the evolutionary development of the latest assembly microsystem which is built around a three fingered, self-aware metrological gripper [1]. Several other processes, machines, and assemblies have been created in the development and support of this machine and are discussed in the context of this micro-scale gripper.

A simplified representation of the design for the three fingered, self-aware metrological gripper is shown in Fig. 1, and an actual picture is shown in Fig. 2. As shown, each finger is attached to a frame, and at each fingertip is a piezoelectric tuning fork oscillator with a slender fiber attached to one tine. On each finger is a set of actuators and optical feedback sensors to provide individual 


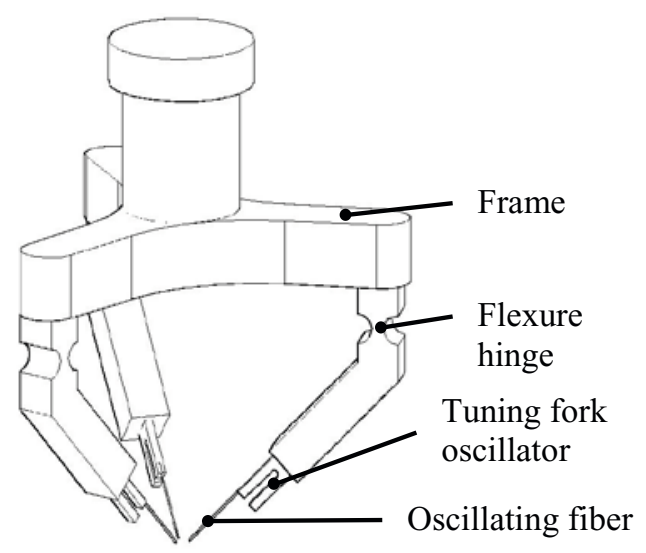

Fig. 1. Simplistic representation of microgripper. Actuation, finger alignments, and feedback sensing mechanisms are not shown. closed-loop synchronization of finger movement in and out of a concentric location (the location of the assembly artifact). Each finger is capable of moving more than 60 $\mu \mathrm{m}$ and sub-micrometer positioning resolution. Translation and the oscillation functions of the tuning fork are controlled using a commercially available, ergonomic video gaming joystick. Hardware comprises data acquisition, video, and joystick hardware with interface software developed in LabVIEWTM, also shown in Fig. 3. Being that the bulk of the mass was in the gripper tool, and the assembly targets are on scales of millimeters or less, it was decided that it would be easier to provide movement of the factory floor in relation to the gripper rather than to provide movement of the gripper in relation to the factory floor. The factory floor, in the form of a commercially available 3 axis translation stage automated for joystick control [1].

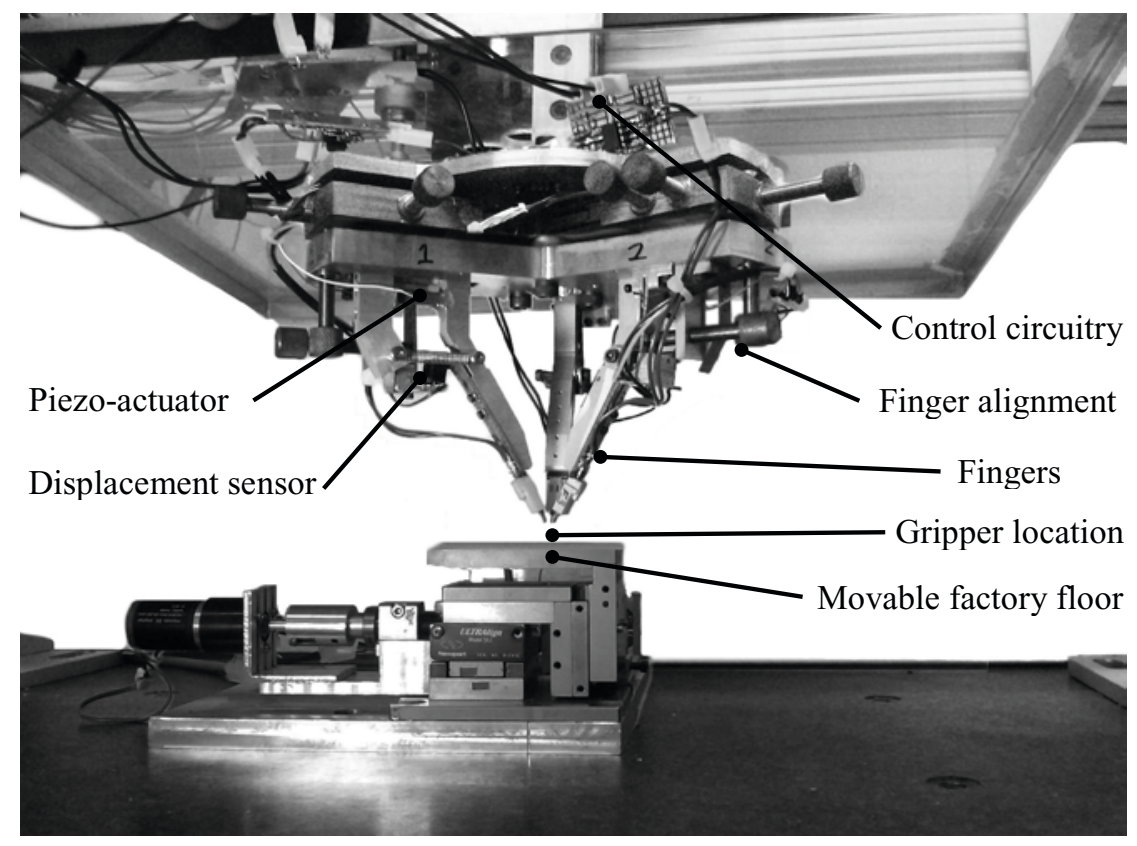

Fig. 2. Snapshot of actual three fingered robot and factory floor 


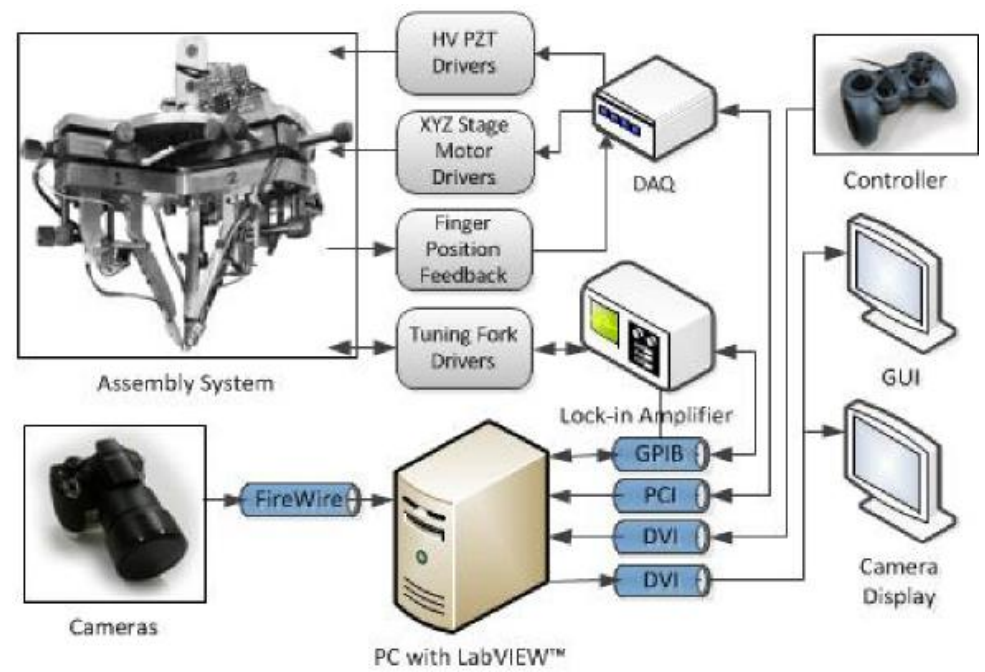

Fig. 3. Block diagram of system hardware implementation

\section{Standing Wave Probe Fingertips}

To provide the necessary dexterity and agility for complex assemblies, the assembly tool generally requires the gripping mechanism, in the region of the component, to have similar, preferably smaller, dimensions. Each of the fingertips of this robotic gripper is, in turn, comprised from a small diameter, high aspect ratio fiber attached to one tine of a piezoelectric, quartz tuning fork. When the dimensions of the fiber are optimized, a standing wave (SW) probe results [2]. In the majority of our studies seven micrometer diameter carbon fibers ranging from three to five millimeters in length were used, typically operating at oscillation frequencies around $32 \mathrm{kHz}$. In some studies fibers with diameters up to $75 \mu \mathrm{m}$ and materials such as glass and tungsten have also been used. These probes provide an innovative micromanipulation technique that enables simultaneous sensing and control of contact forces to ensure reliable assembly operations [3]. Being capable of detecting contact and fingertip position this robotic gripper also has potential for in-situ dimensional measurement of the components of the assembly. The standing wave probes of this study have been researched as a coordinate measuring machine touch sensor and also as a scanning type probe used in surface profilometry $[4,5,6]$. In these studies it has been demonstrated that the dynamic force of the virtual tip (the volume encompassed by the oscillating fiber amplitude) can overcome attraction forces such as meniscus and electrostatic forces that are significant at these scales. In the case of the $7 \mu \mathrm{m}$ diameter carbon fiber with lengths of 3-5 $\mathrm{mm}$, contact forces are estimated to be in the region of micro-Newtons or less [2]. 


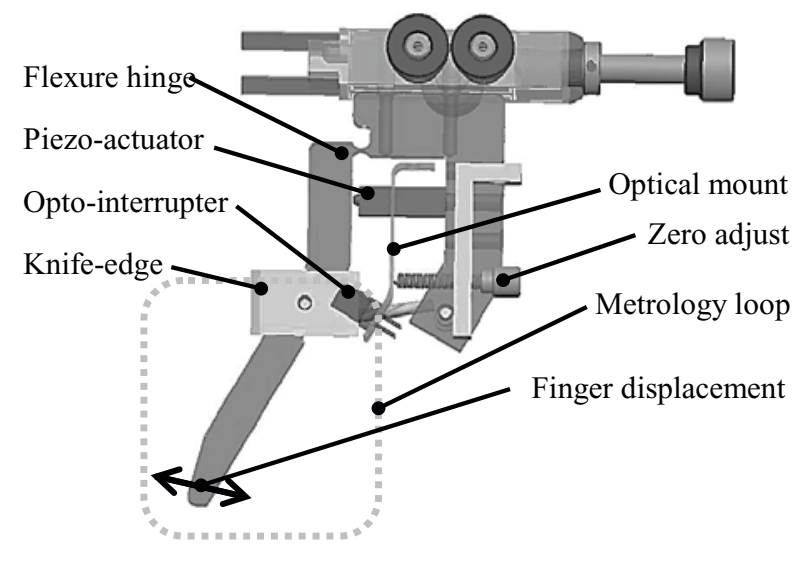

Fig. 4. Solid model of gripper finger showing optointerrupter and knife edge sensor in relation to piezoelectric actuator and path of finger displacement. For clarity, not shown is a spring to apply a restoring force and a preload on the Piezo-actuator.
Additional benefits are that probes in this microgripper application is that the oscillation can be toggled on and off to take advantage of the adhesion forces when desired and to enable release and interaction force sensing of the assembly objects upon measurement and/or intended release. Also, in practice, these SW probes provide significant changes in signal as the virtual tip approaches the surface prior to contact [6].

\section{$3 \quad$ Positioning and Sensing for Fingertip Control}

Fig. 4 shows a solid model of the finger assembly and indicates the metrology within each finger and partial frame of the gripper. Also shown are five of the seven alignment screws making up a total 21 independent adjustments for locating all three fingertips. Also shown is the localized metrology loop within each finger itself. Using a single fingertip as a sensor, this gripper would operate as a metrology tool (CMM or profiling probe) in the same manner, with the same potential performance, as demonstrated in previous research $[4,5,6]$. Contacting probe tips together can be used as a zero reference prior to dimensional measurement. Zeroing prior to each measurement minimizes the effects of drift. With a three finger gripper, three measurement points will be obtained and can be used for dimensional metrology for some complex component shapes and geometric information for components of known form. As shown in Fig. 4, a piezoelectric actuator applies a force against each finger causing a deflection at the flexure hinge that, due to leverage, results in an increased displacement at the fingertip.

To monitor the displacement of the fingers, a knife edge sensor based on an optointerrupter was used [7]. In this case a polished knife edge attaches to a moving finger while the opto-interrupter is mounted into a flexible connecter board with manual adjustment for centering the sensor to accommodate postalignment of the finger position. Such a sensor demonstrates a signal to noise 
ratio (SNR) of 8000 over a displacement rang of $100 \mu \mathrm{m}$ at a bandwidth of $1 \mathrm{kHz}$ and only adds the mass of the knife edge to the mass of the moving finger. A similar method using a laser diode, as an illumination source, coupled to an optical fiber provides much higher power and stability than the LED light source of the opto-interrupter. In this case, the knife edge obstructs the beam across the end of this optical fiber. This latter design has demonstrated an SNR of 40,000 at $1 \mathrm{kHz}$ bandwidth with working ranges between 4 to $90 \mu \mathrm{m}$ [7]. This improved performance capacity will enable future design revisions aimed toward smaller, faster, and more precise assembly tools.

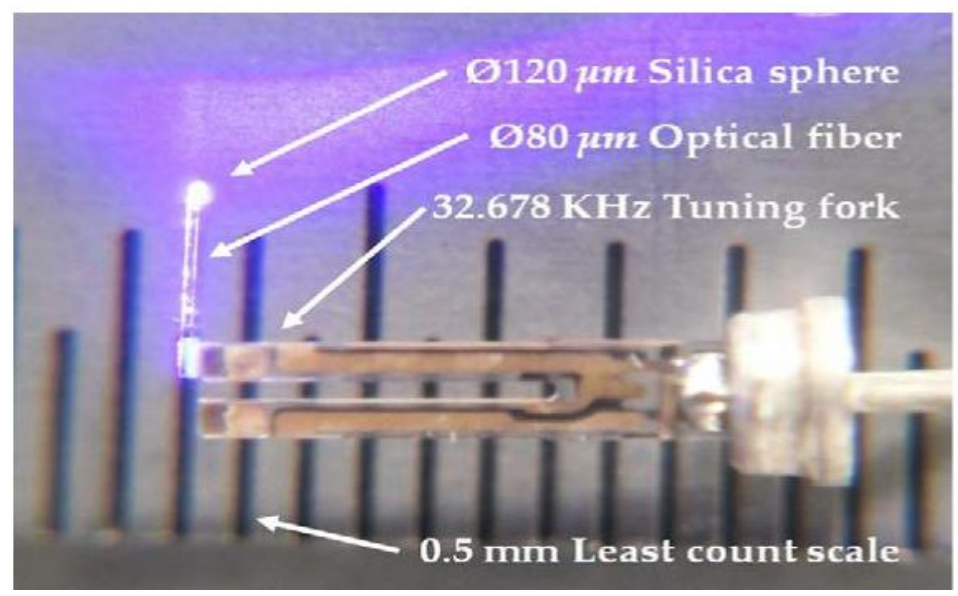

Fig. 5. Micro- CMM probe assembled from a tuning fork, optical fiber and silica sphere illuminated by a laser pointer

\section{$4 \quad$ Electrochemical Etching Facility}

One advantage of the standing wave probes is that they are readily commercially available as CMM probe tips [8]. However because each probe has a high dynamic amplification, Q, and similar, but different, resonant frequency, a method is needed to manufacture each fingertip with an optimal sensitivity at the same frequency and with a similar phase shift. Such probes should provide the ability to excite each tuning fork using the same signal source leading to the potential for synchronizing motion at the finger tips. The idea of this synchronization has many benefits. Simultaneous contact and release, in principal, imposes no net force when balanced by synchronized contact. Being driven by the same signal, switching the oscillator signal will be transmitted simultaneously at all forks to ensure that dynamic changes in fingertip displacements are synchronized. The virtual probe tips in this three finger configuration combine to produce a virtual cage such that components can be gripped while the fingertips are oscillating. In previous studies using single and 
two finger (tweezers) assembly tools, when picking up and transporting components it was not possible to activate the fingers because the component would be either ejected or unpredictably dropped. Additionally, having a single oscillating source reduces circuit complexity and minimizes potential for signal cross-talk issues.

As a method to achieve this necessary fine tuning of SW probes, a system for quantitative electrolytic mass removal from tungsten wires etched in a $\mathrm{KOH}$ electrolyte solution has been developed by our group [9]. This method provides a direct measure of mass removal and is capable of resolving mass changes of less than $40 \mathrm{ng}$. In experiments, a tungsten fiber attached to one tine of the oscillator was incrementally etched to remove $5 \mu \mathrm{g}$ up to $120 \mu \mathrm{g}$ of total mass. In general frequency shifts of $2.8 \mathrm{~Hz} \bullet \mu^{-1}$ were observed with sub-microgram resolution between measured frequency shift and charge-based mass measurement. At the current mass resolution, this corresponds to an ability to control the natural frequency of the finger to within $0.12 \mathrm{~Hz}$ [9].
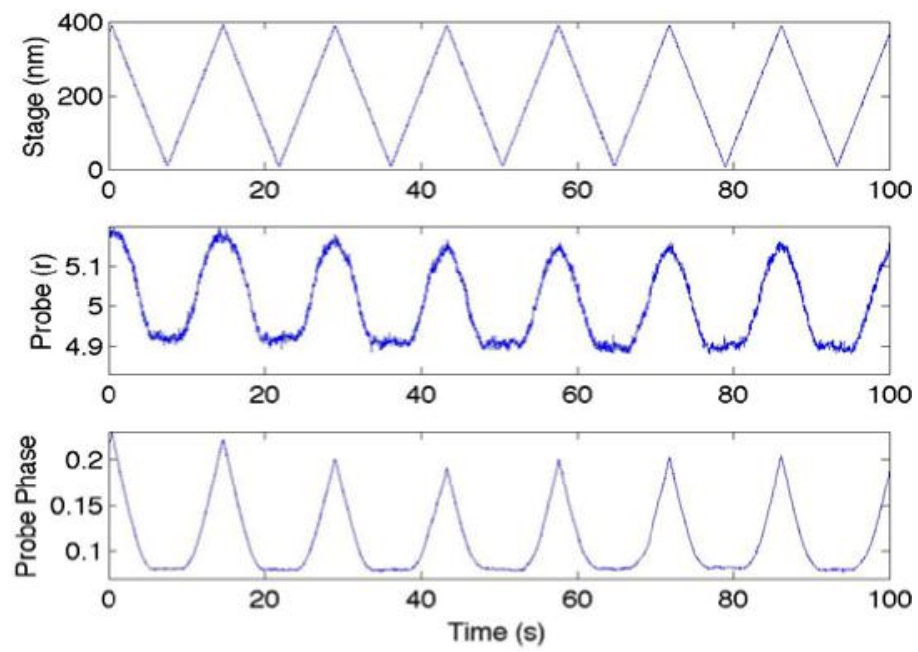

Fig. 6. Measured response of micro-CMM probe. Upper graph shows measured positioner displacement (relative to positioner frame). Middle and lower graphs show magnitude and phase response of micro-CMM probe respectively.

\section{Micro-CMM Probe Assembly}

The foundational goal of this assembly facility was to develop a versatile research tool that would provide an intuitive user interaction and with broad capabilities. Ergonomic controls and graphical and imaging displays have been successfully implemented, thus lowering the learning curve for new operators. Because it is possible to extract proximity, tip position, as well as contact force from measured signals, it is possible to implement haptic feedback into the system. Once implemented, this will enable users to indirectly feel the effects of assembly 
forces further aiding in the development of operator intuition across the microassembly industry.

A major focus in our group is in the field of micro-metrology and the development of new micro sensors. One particular micro CMM probe design was assembled using the three fingered gripper. This probe design utilizes a vibrating mechanical sensor assembled from three components. These components are a 32 $\mathrm{KHz}$ tuning fork, an $80 \mu \mathrm{m}$ diameter fiberglass shaft and a $90 \mu \mathrm{m}$ alumina sphere, all put together with either 50 minute or UV Epoxy. To produce the finished probe the assembly sequence was; the end of the tuning fork tine was positioned in the stationary focal plane of the camera, the optical fiber was clamped vertically on the XYZ factory floor, glue was applied to the end of the tuning fork tine, the factory floor was then used to contact the optical fiber to the end of the tuning fork tine and the glue cured, the sphere and tuning fork with attached optical fiber was then placed onto the moving factory floor, glue was applied to the end of the optical fiber, the sphere was picked up with the three finger gripper and placed on the optical fiber. A photograph of an assembled probe is shown in Fig. 5.

To evaluate performance of the probe, a nano-positioning stage was used to reciprocate a specimen over a range of $400 \mathrm{~nm}$ with the surface of this specimen contacting the probe near midrange. Each cycle comprised a linear motion toward and away from the probe tip so that position of the surface plotted against time produced the triangular plot shot in the upper graph of Fig. 6. The two plots below this show the magnitude and phase response of the micro-CMM probe using a Stanford SRS850 Lock-in amplifier. These graphs show a relatively flat response prior to contact while during contact the signal changes follow that of the specimen displacement.

From the response curves in Fig. 6 it is clear that the phase signal is more linear and has lower SNR to that of the amplitude response. Discussion of probe performance is beyond the scope of this paper. However it is clear that this microCMM probe is capable of detecting mechanical interactions with resolution of better than $15 \mathrm{~nm}$. Ideally this current assembly tool might contribute to the micro-manufacture of scaled down tools leading to future nano-assembly machines.

\section{References}

1. Hastings, D.J., Graham, J., Nowakowski, B., Smith, S.T., Tomblin, J.: A three fingered hand for a micro-assembly system. In: Proc. ASPE, vol. 50, pp. 93-96 (2010)

2. Bauza, M., Woody, S.C., Hocken, R.J., Smith, S.T.: Development of a virtual probe tip with an application to high aspect ratio microscale features. Rev. Sci. Instrum. 76(9), 95112 (8 pages) (2005)

3. Woody, S.C., Nowakowski, B., Bauza, M., Smith, S.T.: Standing wave probes for microassembly. Rev. Sci. Instrum. 79, 85107 (9 pages) (2008)

4. Bauza, M.B., Woody, S.C., Woody, B.A., Smith, S.T.: Surface profilometry of high aspect ratio features. Wear 271, 519-522 (2011) 
5. Bauza, M., Woody, S.C., Smith, S.T., Woody, B.A.: Microscale surface and form profilometry using a standing wave probe. In: Metromet Conference, Bilboa, Spain (February 2010)

6. Bauza, M., Woody, S.C., Seugling, R.M., Smith, S.T.: Dimensional measurements of ultra delicate materials using micrometrology tactile sensing. In: Proc. ASPE, vol. 50, pp. 73-76 (2010)

7. Lin, F., Smith, S.T., Ghazanfar, H.: Knife edge position transducer performance studies, Precision Engineering (submitted, 2011)

8. Insitutec Inc., 45 Odell School Road Suite A, Concord, North Carolina 28027, insitutec.com.

9. Nowakowski, B., Smith, S.T., Pratt, J., Shaw, G.: Electrolysis for precision mass removal. Rev. Sci. Instrum. (to be submitted, 2011) 